\title{
The Health Burden of Cancer Attributable to Obesity in Korea: A Population-Based Cohort Study
}

\author{
Joo Eun Lee, $\mathrm{PhD}{ }^{1}$ \\ Chung Mo Nam, MD, PhD² \\ Sang Gyu Lee, MD, $P h D^{3,4}$ \\ Sohee Park, PhD,5 \\ Tae Hyun Kim, PhD 3,4 \\ Eun-Cheol Park, MD, PhD 2,3
}

\begin{abstract}
Purpose
Considering the health impact of obesity and cancer, it is important to estimate the burden of cancer attributable to high body mass index (BMI). Therefore, the present study attempts to measure the health burden of cancer attributable to excess BMI, according to cancer sites.

\section{Materials and Methods}

The present study used nationwide medical check-up sample cohort data (2002-2015). The study subjects were 496,390 individuals (268,944 men and 227,446 women). We first calculated hazard ratio (HR) in order to evaluate the effect of excess BMI on cancer incidence and mortality. Then, the adjusted HR values and the prevalence of excess BMI were used to calculate the population attributable risk. This study also used the Global Burden of Disease method, to examine the health burden of obesity-related cancers attributable to obesity.
\end{abstract}

\section{Results}

The highest disability-adjusted life year (DALY) values attributable to overweight and obesity in men were shown in liver cancer, colorectal cancer, and gallbladder cancer. Among women, colorectal, ovarian, and breast (postmenopausal) cancers had the highest DALYs values attributable to overweight and obesity. Approximately $8.0 \%$ and $12.5 \%$ of cancer health burden (as measured by DALY values) among obesity-related cancers in men and women, respectively, can be prevented.

\section{Conclusion}

Obesity has added to the health burden of cancer. By measuring the proportion of cancer burden attributable to excess BMI, the current findings provide support for the importance of properly allocating healthcare resources and for developing cancer prevention strategies to reduce the future burden of cancer.

\section{Key words}

Neoplasms, Obesity, Body mass index, Health burden, Disability-adjusted life years

\section{Introduction}

Cancer a major cause of mortality and morbidity worldwide and is expected to increase sharply with global aging and increasing cancer incidence [1]. Cancer is still the primary cause of death since 1983, with about one million cancer patients and with about 220,000 new cancer cases and 74,000 cancer deaths annually [2,3]. Furthermore, according to the International Agency for Research on Cancer's GLOBOCAN report, Korea's 5-year cancer prevalence rate (1,523 per 100,000$)$ is more than twice the international prevalence rate $(625$ per 100,000) [4]. Population aging, progress of treatment, and cancer screening programs are increasing both the incidence and the survival rate of cancer [5].

When targeting a particular population, the burden of disease can be measured as the difference between an ideal health level and the actual health level. The health burden of 
disease can be measured by several indicators, including mortality, incidence, prevalence, health-adjusted life expectancy (HALE), disability-adjusted life years (DALYs), quality-adjusted life years (QALYs), and Healthy Life years (HeaLYs) [6]. Mortality, incidence, and prevalence are the most basic indices, but these indicators do not include information about quality of life. These traditional indicators of disease burden do not reflect improvements in health conditions since the illness. Another way to measure the burden of disease is to measure the burden of disease with a health index that takes into account quality of life (QOL), which is measured as an indicator of disease status and death combined. The World Health Organization (WHO) and the Global Burden of Disease (GBD) Study Group developed the DALYs indicator in order to reflect both time of disability and time lost due to premature death [7]. The reason for estimating the burden of disease using various methods is that the measurement of the burden of disease helps to improve health care decision-making by improving the allocation of healthcare resources and the assessment of the effects of health-related interventions.

The WHO has defined obesity as a disease and has predicted that it will become a health problem as important as smoking in the 21st century, with a 2014 survey showing that the worldwide obesity prevalence in 2014 had doubled compared to in 1980 [8]. The prevalence of obesity in Korea has increased from $26.2 \%$ of men and $25.1 \%$ of women in 1998 to $34.7 \%$ of men and $27.3 \%$ of women in 2005, and $35 \%-39 \%$ of men and $23 \%-28 \%$ of women have been maintained until recently.

There have been many studies on obesity and cancer in Korea [9-11]. Most studies have estimated the burden of cancer using cancer incidence or mortality, which do not reflect patients' QOL [12-14]. On the other hand, we used DALYs to evaluate the health burden of cancer which reflects patients' QOL. DALYs evaluate health differences that combine life lost due to disability and premature death. Comprehensive information using DALY can help to quantify the burden of obesity-related cancer and allocate health care resources. In addition, most studies that have estimated the relative risk of cancer given the presence of obesity have not reflected other confounding variables [14,15]. Furthermore, studies about obesity and cancer risk have not reflected recent changes in the prevalence of obesity and the increasing epidemiological changes in cancer risk. The most recent representative large cohort study in Korea, the postmenopausal Korean women study and the Korean Cancer Prevention Study, were followed up to 2003 and 2006, respectively. Since then, there has been no representative cohort study of obesity and cancer burden in recent years [16-18]. Therefore, the present study aims to examine the health burden of obesityrelated cancers attributable excess body mass index (BMI) by using nationwide cohort sample data using the GBD method. Considering the health impact of obesity and cancer, it is important to measure the burden of cancer, taking into account recent epidemiological changes.

\section{Materials and Methods}

\section{Study population and design}

This study used medical check-up nationwide sample cohort data from National Health Insurance Service (NHIS) claims in 2002 and 2015. The medical check-up cohort data included demographic, socioeconomic, and mortality-related information as well as information about medical utilization, hospital characteristics, and health check-ups. The data included approximately $10 \%$ of all participants who received a general health check-up as of 2002-2003, aged 40-79 in 2002, a total of 514,866 Koreans (279,125 men and 235,741 women). This study used 514,866 populations who received a general health check-up in 2002-2003, aged 40-79. Of these, the participants who were diagnosed with cancer $(n=17,335)$ or died $(\mathrm{n}=686)$ during 2002-2003 were excluded. In addition, we excluded participants whose BMI data at baseline were missing $(n=455)$. Ultimately, the study population comprised 496,390 individuals (268,944 men and 227,446 women).

\section{Variables}

To analyze the health burden of cancer attributable to overweight or obesity, we examined DALYs of obesity-related cancers attributable to excess BMI as outcome variables (Table 1). The DALY measure includes both the years of life lost (YLL) to premature death and years lived with disability (YLD). To quantify the burden of cancer patients, the WHO and the GBD developed DALY. It measures the difference between current health and ideal status as an index of years of healthy life lost. Disability weights, discounting, and ageweighting of DALYs were used in this study.

The development of cancer was defined according to the first cancer admission event using the 10th version of the International Classification of Disease codes in admission records, and cancer mortality was defined as death by cancer using cause of death information in Statistics Korea, after excluding cancer cases and deaths during first 2 years. In addition, obesity-related cancer types were identified by the International Agency for Research on Cancer (IARC), the World Cancer Research Fund (WCRF), and the American Institute for Cancer Research (AICR) [19-21]. There is sufficient evidence that overweight or obesity increases the risk 
of esophageal, colorectal, pancreatic, post-menopausal breast, endometrial, kidney, and liver cancers. On the other hand, cancer sites with limited evidence for a link to obesity include gallbladder, ovarian, and advanced prostate cancers (S1 Table).

The primary interesting variable is BMI. The most frequently used measure of underweight, overweight, and obese is BMI. It was calculated as height and weight obtained from medical check-up (weight $[\mathrm{kg}] /$ height $\left[\mathrm{m}^{2}\right]$ ) and categorized as underweight (BMI $\left.<18.5 \mathrm{~kg} / \mathrm{m}^{2}\right)$, normal (18.5$\left.22.9 \mathrm{~kg} / \mathrm{m}^{2}\right)$, overweight $\left(23-24.9 \mathrm{~kg} / \mathrm{m}^{2}\right)$, obese (25-29.9 kg/ $\left.\mathrm{m}^{2}\right)$, and severely obese $\left(\geq 30 \mathrm{~kg} / \mathrm{m}^{2}\right)$. Cancer risk factors including BMI used were not updated from baseline medical check-up in this analysis.

Other independent variables are demographic and socioeconomic variables (sex, age, income level), health check-up variables (smoking status, drinking, physical activity, family history of cancer, total cholesterol), and medical utilization variables (Charlson comorbidity index [CCI], regular use of aspirin). Smoking status was classified as never smoker, former smoker, and current smoker. Alcohol consumption was categorized as non-drinker (no alcohol consumption), moderate drinker (up to 14 drinks for men and up to 7 drinks for women per week), or heavy drinker (15 drinks or more for men and 8 drinks or more for women per week). Physical activity was categorized as never, less than 3 days per week, 3 to less than 5 days per week, or more than 5 days per week. CCI score is helpful to predict 1-year mortality. The comorbid condition is assigned to 1, 2, 3, or 6 depending on comorbidity's severity. Several studies have shown that the use of aspirin is associated with reduced risk of overall cancer
$[22,23]$. The U.S. Preventive Services Task Force recommended the use of aspirin for primary prevention of colorectal cancer [24]. Regular use of aspirin was defined as aspirin use at least 2 times per week. Family history of cancer was asked with biennial questionnaire. Only gynecological cancer was adjusted for oral contraceptive (OC) and hormone replacement therapy (HRT) use. In case of OC and HRT, because the risk of cancer decreases rapidly after cessation of use, we used OC and HRT of one year prior to the onset of cancer [25].

\section{Statistical analysis}

Chi-square and t test were performed to assess the differences in characteristics according to cancer incidence and mortality. To calculate the population attributable risk (PAR), the basic formula is the following Levin's formula:

$$
\operatorname{PAR}=\frac{\sum p_{i}\left(R R_{i}-1\right)}{\sum p_{i}\left(R R_{i}-1\right)+1}
$$

, where $R R_{i}$ is the relative risk and is $p_{i}$ prevalence of risk factor.

On the other hand, as BMI category is divided into five categories, more sophisticated method is regarded to calculate PAR of excess BMI as follows:

$$
\operatorname{PAR}=\frac{\sum p_{o w}\left(R R_{o w}-1\right)+\sum p_{o}\left(R R_{o}-1\right)+\sum p_{s o}\left(R R_{s o}-1\right)}{\sum p_{o w}\left(R R_{o w}-1\right)+\sum p_{o}\left(R R_{\sigma}-1\right)+\sum p_{s o}\left(R R_{s o}-1\right)+1}
$$

, where $R R_{o w}, R R_{o}$, and $R R_{\text {so }}$ are the adjusted-relative risk of cancer for overweight, obesity, and severe obesity respec-

Table 1. Data sources and formulas for DALYs

YLL

$\mathrm{YLL}=M \times\left\{\left[K C e^{r a} /(r+\beta)^{2}\right]\left[e^{-(r+\beta)(L+a)}[-(r+\beta)(L+a)-1]-e^{-(r+\beta) a}[-(r+\beta) a-1]\right]+[(1-K) / r]\left(1-e^{-r L}\right)\right\}$

Average age at onset for each age cohort (a)

Discount rate $(\mathrm{r}=0.03)$

Age-weighting constants $(\beta=0.04, K=1.00, C=0.1658)$

Average age

Mortalities (M)

Standard life expectancy at the age of death (L)

\section{YLD}

$\mathrm{YLD}=I \times D W \times\left\{\left[K C e^{r a} /(r+\beta)^{2}\right]\left[e^{-(r+\beta)(L+a)}[-(r+\beta)(L+a)-1]-e^{-(r+\beta) a}[-(r+\beta) a-1]\right]+[(1-K) / r]\left(1-e^{-r L}\right)\right\}$

Incidences (I)

Disability weights (DW)

Average age at onset for each age cohort (a)

Discount rate $(\mathrm{r}=0.03)$

Age-weighting constants $(\beta=0.04, K=1.00, C=0.1658$ )

Expected duration of disability (L)

YLL, years of life lost; YLD, years lived with disability; DALY, disability-adjusted life year. 

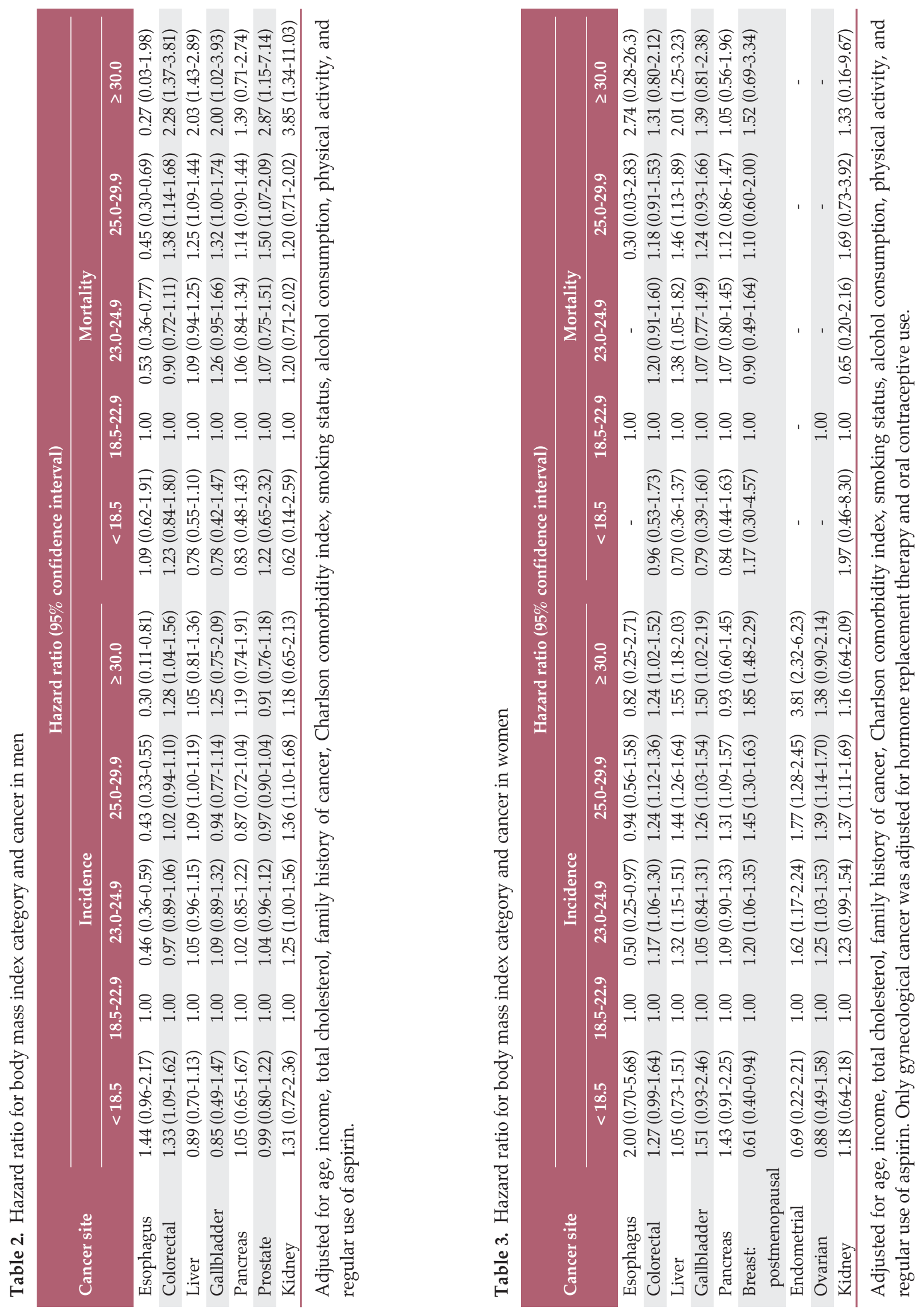
tively, and $p_{o w}, p_{0}$, and $p_{s 0}$ are prevalence of overweight, obesity, and severe obesity respectively. To estimate the adjustedrelative risk, we first performed Cox proportional hazard model to evaluate the effect of excess BMI on cancer incidence and mortality, adjusting confounding variables and stratifying sex and cancer type.

To evaluate the health burden of cancer patients attributable to obesity in Korea, we estimated DALYs as the sum of the YLL and YLD. To estimate YLL, the cancer mortality and standard life expectancy were used. To estimate YLD, cancer incidence, average duration of cancer, and disability weight were used (Table 1).

\section{Ethical statement}

This study was approved by the Institutional Review Board of Yonsei University Severance Hospital (Y-20170032). The informed consent was waived.

\section{Results}

The study population characteristics at baseline (20022003) are shown in S2 Table. Table 2 shows hazard ratio [HR] for BMI category and obesity related-cancer sites which is confirmed by IARC, WCRF and AICR. Among cancer types examined in this study, colorectal and kidney cancers had significantly high $\mathrm{HR}$ estimates for cancer incidence in men (colorectal, BMI 25.0-29.9 kg/m²: HR, 1.02; 95\% confidence interval [CI], 0.94 to $1.10 ; \mathrm{BMI} \geq 30.0 \mathrm{~kg} / \mathrm{m}^{2}$ : $\mathrm{HR}, 1.28 ; 95 \%$ CI, 1.04 to 1.56 ; kidney, BMI $23.0-24.9 \mathrm{~kg} / \mathrm{m}^{2}$ : HR, $1.25 ; 95 \%$ CI, 1.00 to 1.56; BMI $25.0-29.9 \mathrm{~kg} / \mathrm{m}^{2}: \mathrm{HR}, 1.36 ; 95 \%$ CI, 1.10 to 1.68). Obesity was significantly associated with risk for mortality from kidney, prostate, colorectal, liver, and gallbladder cancers (kidney, BMI $\geq 30.0 \mathrm{~kg} / \mathrm{m}^{2}: \mathrm{HR}, 3.85 ; 95 \%$ CI, 1.34 to 11.03; prostate, BMI 25.0-29.9 kg/ m²: HR, 1.50; $95 \%$ CI, 1.07 to $2.09 ; \mathrm{BMI} \geq 30.0 \mathrm{~kg} / \mathrm{m}^{2}: \mathrm{HR}, 2.87 ; 95 \% \mathrm{CI}, 1.15$ to 7.14; colorectal, BMI 25.0-29.9 kg/m²: HR, 1.38; 95\% CI, 1.14 to $1.68 ; \mathrm{BMI} \geq 30.0 \mathrm{~kg} / \mathrm{m}^{2}$ : HR, $2.28 ; 95 \% \mathrm{CI}, 1.37$ to 3.81 ; liver, BMI $25.0-29.9 \mathrm{~kg} / \mathrm{m}^{2}$ : HR, $1.25 ; 95 \%$ CI, 1.09 to $1.44 ;$ BMI $\geq 30.0 \mathrm{~kg} / \mathrm{m}^{2}: \mathrm{HR}, 2.03 ; 95 \%$ CI, 1.43 to 2.89; gallbladder, BMI $25.0-29.9 \mathrm{~kg} / \mathrm{m}^{2}$ : HR, 1.32; 95\% CI, 1.00 to $1.74 ; \mathrm{BMI} \geq 30.0$ $\mathrm{kg} / \mathrm{m}^{2}: \mathrm{HR}, 2.00 ; 95 \%$ CI, 1.02 to 3.93 ).

HR for BMI category and cancer in women is shown in Table 3. Endometrial cancer had the highest HR estimate for cancer incidence in women (BMI 25.0-29.9 kg/m²: HR, 1.77; $95 \%$ CI, 1.28 to $2.45 ; \mathrm{BMI} \geq 30.0 \mathrm{~kg} / \mathrm{m}^{2}: \mathrm{HR}, 3.81 ; 95 \%$ CI, 2.32 to 6.23). Other cancer sites with significant HR estimates for cancer incidence were breast (postmenopausal), liver, gallbladder, colorectal, ovary, kidney, pancreas in women (breast, $\mathrm{BMI} \geq 30.0 \mathrm{~kg} / \mathrm{m}^{2}: \mathrm{HR}, 1.85 ; 95 \% \mathrm{CI}, 1.48$ to 2.29 ; liver, BMI $25.0-29.9 \mathrm{~kg} / \mathrm{m}^{2}$ : HR, $1.44 ; 95 \% \mathrm{CI}, 1.26$ to $1.64 ; \mathrm{BMI} \geq 30.0$ $\mathrm{kg} / \mathrm{m}^{2}: \mathrm{HR}, 1.55$; $95 \%$ CI, 1.18 to 2.03; gallbladder, BMI 25.0$29.9 \mathrm{~kg} / \mathrm{m}^{2}: \mathrm{HR}, 1.26 ; 95 \% \mathrm{CI}, 1.03$ to $1.54 ; \mathrm{BMI} \geq 30.0 \mathrm{~kg} / \mathrm{m}^{2}$ : HR, 1.50; 95\% CI, 1.02 to 2.19; colorectal, BMI 25.0-29.9 $\mathrm{kg} / \mathrm{m}^{2}: \mathrm{HR}, 1.24 ; 95 \% \mathrm{CI}, 1.12$ to $1.36 ; \mathrm{BMI} \geq 30.0 \mathrm{~kg} / \mathrm{m}^{2}: \mathrm{HR}$, 1.24; $95 \%$ CI, 1.02 to 1.52 ; ovary, BMI $25.0-29.9 \mathrm{~kg} / \mathrm{m}^{2}: \mathrm{HR}$, 1.39; $95 \%$ CI, 1.14 to 1.70; kidney, BMI $25.0-29.9 \mathrm{~kg} / \mathrm{m}^{2}: \mathrm{HR}$, 1.37; $95 \%$ CI, 1.11 to 1.69 ; pancreas, BMI $25.0-29.9 \mathrm{~kg} / \mathrm{m}^{2}: \mathrm{HR}$, $1.31 ; 95 \% \mathrm{CI}, 1.09$ to 1.57). Liver cancer had a significant HR estimate for cancer mortality in women (BMI $25.0-29.9 \mathrm{~kg} /$ $\mathrm{m}^{2}: \mathrm{HR}, 1.46 ; 95 \% \mathrm{CI}, 1.13$ to $1.89 ; \mathrm{BMI} \geq 30.0 \mathrm{~kg} / \mathrm{m}^{2}: \mathrm{HR}, 2.01$; $95 \%$ CI, 1.25 to 3.23 ).

The prevalence of BMI category according to sex and age group is shown in S3 Table. Table 4 shows the population attributable fraction (PAF) of obesity by cancer types. Over-

Table 4. Estimated number of cancer cases and deaths attributable to overweight and obesity

\begin{tabular}{|c|c|c|c|c|c|c|c|c|}
\hline & \multicolumn{4}{|c|}{ Men } & \multicolumn{4}{|c|}{ Women } \\
\hline & PAF (\%) & Cases & PAF (\%) & Deaths & PAF $(\%)$ & Cases & PAF $(\%)$ & Deaths \\
\hline Colorectal & 1.4 & 78 & 13.7 & 123 & 11.5 & 364 & 11.0 & 48 \\
\hline Liver & 4.5 & 201 & 11.9 & 204 & 19.5 & 332 & 22.2 & 100 \\
\hline Gallbladder & 3.0 & 27 & 17.1 & 77 & 10.3 & 71 & 10.1 & 33 \\
\hline Pancreas & 1.0 & 11 & 7.0 & 46 & 11.0 & 91 & 5.9 & 23 \\
\hline Breast: postmenopausal & & & & & 18.6 & 554 & 5.1 & 9 \\
\hline Endometrial & & & & & 34.0 & 101 & - & - \\
\hline Ovary & & & & & 17.0 & 132 & - & - \\
\hline Prostate & 1.2 & 78 & 18.7 & 54 & & & & \\
\hline Kidney & 16.4 & 133 & 15.8 & 21 & 15.5 & 59 & 18.7 & 8 \\
\hline$\%$ of all cancers & 1.4 & & 5.5 & & 7.1 & & 6.3 & \\
\hline
\end{tabular}

$\mathrm{PAF}$, population attributable fraction. 


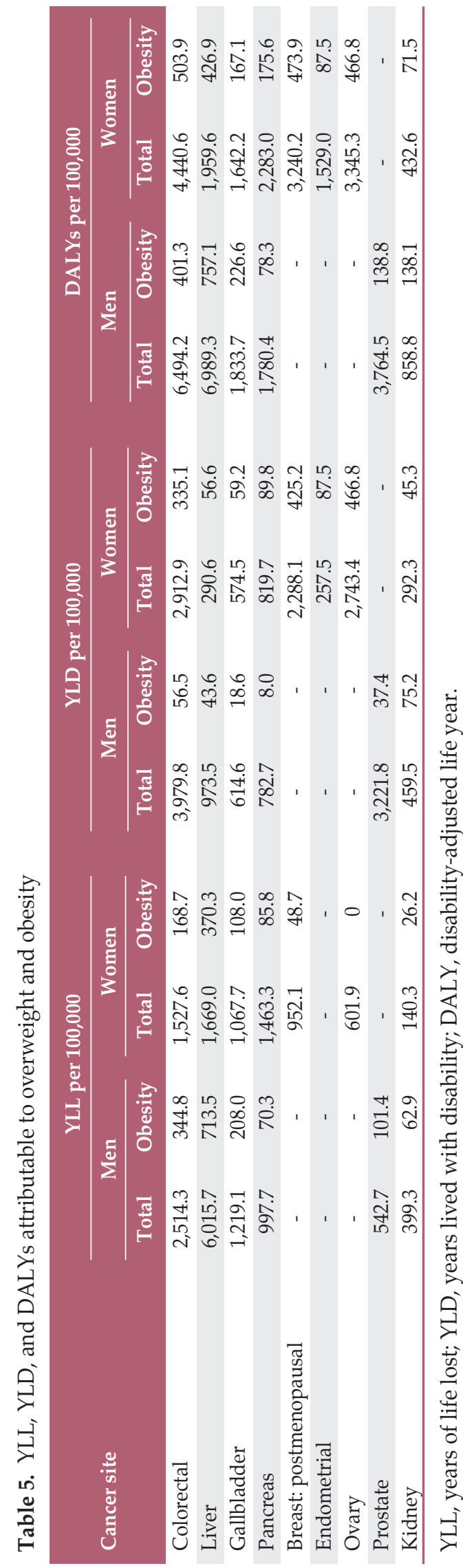

weight and obesity were responsible for 528 cancer incidences $(1.4 \%)$ and 525 cancer deaths $(5.5 \%)$ in men. Among women, 1,704 cancer incidences $(7.1 \%)$ and 222 cancer deaths $(6.3 \%)$ were attributable to overweight and obesity.

Table 5 shows YLL, YLD, and DALY attributable to overweight and obesity by cancer site, during the follow-up period. DALYs of cancer attributable to overweight and obesity were 1,740 person-years per $100,000(8.0 \%)$ in men and 2,373 person-years per $100,000(12.5 \%)$ in women. The cancer sites with the highest DALY values among men were liver, colorectal, and prostate cancers (liver, 6,989.3 person-years per 100,000; colorectal, 6,494.2 person-years per 100,000; prostate, $3,764.5$ person-years per 100,000). Among women, colorectal, ovarian, and breast (postmenopausal) cancers had the highest DALY values (colorectal, 4,440.6 person-years per 100,000; ovary, 3,345.3 person-years per 100,000; breast, $3,240.2$ person-years per 100,000 ).

\section{Discussion}

To evaluate the burden of cancer attributable to excess BMI in Korea, we estimated the DALYs by obesity-related cancer types identified by IARC, WCRF, and AICR. According to the results of the study, colorectal and kidney cancers had the highest HR estimates for cancer incidence in men. Endometrial, post-menopausal breast, liver, gallbladder, colorectal, ovarian, kidney, and pancreas cancers had significant HR estimates for cancer incidence in women. Similar to the cancer statistics from the Korea Central Cancer Registry (KCCR) in 2014, in this study the age-standardized cancer incidence rate among the obesity-related cancer types was highest in colorectal and liver cancers among men and in breast and colorectal cancers among women [29]. With regard to the age-standardized cancer mortality rate, according to KCCR liver and colorectal cancers among men and colorectal, liver, and breast cancers among women were highest. Although the absolute values were different because of different subjects and units, the results were similar when comparing this study's ranks for cancer types with those of KCCR.

The findings of this study were similar to those of previous studies concerning obesity and cancer risk. Reeves et al. (2007) [30] was conducted on British women recruited into the Million Women Study, aged 50-64 in 1996-2001, and they were followed until 2004. Endometrial cancer, adenocarcinoma of the esophagus, and kidney, pancreatic, ovarian, breast, and colorectal cancers had significant HR estimates for cancer incidence in postmenopausal women. The results of the current study and those of the Million Women Study 
showed similar trends, except that HR values of pancreas and kidney cancer were different because the number of severely obese people was small in this study. Jee et al. (2008) [17] analyzed the HR of BMI and cancer in Korean men and women, aged 30-95, who participated in National Health Insurance Corporation (NHIC) health examinations in 19921995, following the study population until 2006. High BMI was significantly associated with risk for colon, liver, and gallbladder cancers in men and for liver, gallbladder, pancreatic, and postmenopausal breast cancers in women. Song et al. (2008) [16] examined BMI and cancer using a cohort of postmenopausal Korean women who participated in NHIC medical evaluations from 1993 to 1994; they were followed up until 2003. Colorectal, gallbladder, breast, corpus uteri, and kidney cancers had significant HR estimates for cancer incidence in postmenopausal women.

The results of this study show the health burden of obesity-related cancer attributable to obesity. Compared with previous studies about obesity-related cancer health burden, the PAF values of this study are lower than in other studies. But careful interpretation of direct comparison with other research results is required, because there are differences between the studies of obesity-related cancer types included in the analysis. An extensive study in Europe showed that the overall proportions of cancer attributable to excess BMI in European were 3\% in men and 6\% in women, while those of the current study were $1.4 \%$ and $7.1 \%$, respectively [31]. The attributable proportions by countries were $2.1 \%$ for Greece men, $4.9 \%$ for Germany men, 3.9\% for Denmark women, and $8.8 \%$ for Spain women. The attributable proportions by cancer type in men and women were $4.4 \%$ for prostate, $8.5 \%$ for breast, $39.2 \%$ for endometrium, $25.5 \%$ and $24.5 \%$ for kidney, and gallbladder $24.8 \%$ and $23.7 \%$, respectively. Compared to the European, proportions attributable to excess BMI were lower for prostate, endometrium, kidney, and gallbladder (prostate, $1.2 \%$; endometrium, $34.0 \%$; kidney, $16.4 \%$ in men, $15.5 \%$ in women; gallbladder, $3.0 \%$ in men, $10.3 \%$ in women) and higher for breast $(18.6 \%)$ in the current study. WCRF reported that the PAF of high BMI in cancer incidence in 2012 were 20.8\% for North America, 17.2\% for Western Europe, and 6.0\% for East Asia among men; however, in the results of the current study the figure was $1.4 \%$ for Korean men [32]. With regard to women, the figures were 14.2\% for North America, 14.5\% for Western Europe, and $8.3 \%$ for East Asia, respectively; on the other hand, in the results of the current study the figure for Korean women was $7.1 \%$. Because the age ranges of subjects were different, the estimates might be different when comparing the attributable burden by cancer types as found in this study with the results of other studies. In addition, the differences of the current study results which were greater from Western countries than from Asian countries might be expected, as the risks of several obesity related-cancers are different by ethnic groups and obesity prevalence in Asian populations is lower than that in Western populations [13,32].

The present study has several limitations. First, the HR values may be uncertain because the HR values were analyzed for $10 \%$ of the participants who received a health checkup in the specified year, not the HR values for the whole population. However, this study used large scale and representative cohort data with confounding variables such as income level, smoking status, alcohol drinking, physical activity, family history of cancer, total cholesterol, comorbidity, and regular use of aspirin. Adjusting these confounders could reduce the uncertainty of the relative risk value. Second, this study used BMI determined during the baseline health check-up and did not update the BMI. It is known that it takes about 10-20 years between exposure and cancer development. However, since this cohort data contains 14-year follow-up data, BMI changes after baseline were not available. Nevertheless, BMI was calculated by height and weight measured in health screenings, not by self-reported height and weight. Third, as the data did not contain information about cancer stages, this study could not consider cancer severity, which affects disability and medical expenses. Sixth, the study also lacks data about postmenopausal status. Nevertheless, since the association between obesity and breast cancer varies according to menopausal status, women over 55 years of age were considered postmenopausal women for the purposes of this study. The median age of menopause in Korean women is 50 , and $95 \%$ are menopausal before the age of 55 [33].

This study found that the DALYs of cancer due to obesity were 2,373.2 person-years per 100,000 in men and 1,740.2 person-years per 100,000 in women, accounting for $8.0 \%$ and $12.5 \%$, respectively, of the health burden of obesity-related cancers. Considering that obesity increases the burden of cancer and the prevalence of obesity is increasing, further efforts are needed to reduce obesity rates. These updated results about the health impact of obesity on cancer provide useful data to help determine how best to allocate healthcare resources and how best to develop cancer prevention strategies to reduce the burden of cancer.

\section{Electronic Supplementary Material}

Supplementary materials are available at Cancer Research and Treatment website (https://www.e-crt.org).

\section{Conflicts of Interest}

Conflict of interest relevant to this article was not reported. 


\section{References}

1. Yancik R. Population aging and cancer: a cross-national concern. Cancer J. 2005;11:437-41.

2. Statistics Korea. Cause of death statistics. Daejeon: Statistics Korea; 2015.

3. Jung KW, Won YJ, Kong HJ, Lee ES. Cancer statistics in Korea: incidence, mortality, survival, and prevalence in 2015. Cancer Res Treat. 2018;50:303-16.

4. Ferlay J, Soerjomataram I, Ervik M, Dikshit R, Eser S, Mathers C, et al. GLOBOCAN 2012: Estimated cancer incidence, mortality, and prevalence worldwide in 2012. Lyon: IARC; 2012.

5. Suh M, Song S, Cho HN, Park B, Jun JK, Choi E, et al. Trends in participation rates for the national cancer screening program in Korea, 2002-2012. Cancer Res Treat. 2017;49:798-806.

6. Murray CJ, Salomon JA, Mathers CD, Lopez AD; World Health Organization. Summary measures of population health: concepts, ethics, measurement and applications. Geneva: World Health Organization; 2002.

7. Murray CJ, Lopez AD. Alternative projections of mortality and disability by cause 1990-2020: Global Burden of Disease Study. Lancet. 1997;349:1498-504.

8. World Health Organization. Obesity: preventing and managing the global epidemic. Report of a WHO Consultation (WHO Technical Report Series 894). Geneva: World Health Organization; 2000.

9. Park SM, Yun YH, Kim YA, Jo M, Won YJ, Back JH, et al. Prediagnosis body mass index and risk of secondary primary cancer in male cancer survivors: a large cohort study. J Clin Oncol. 2016;34:4116-24.

10. Shin HY, Jee YH, Cho ER. Body mass index and incidence of thyroid cancer in Korea: the Korean Cancer Prevention StudyII. J Cancer Res Clin Oncol. 2017;143:143-9.

11. Shin CM, Lee DH, Choi YJ, Park YS, Yoon H. The association between obesity, metabolic health, and the risk for colorectal cancer in the general population in Korea using National Health Insurance Service: national sample cohort (NHISNSC). Gastroenterology. 2017;152(5 Suppl 1):S880.

12. Calle EE, Rodriguez C, Walker-Thurmond K, Thun MJ. Overweight, obesity, and mortality from cancer in a prospectively studied cohort of U.S. adults. N Engl J Med. 2003;348:1625-38.

13. Renehan AG, Tyson M, Egger M, Heller RF, Zwahlen M. Body-mass index and incidence of cancer: a systematic review and meta-analysis of prospective observational studies. Lancet. 2008;371:569-78.

14. Parkin DM, Boyd L. 8. Cancers attributable to overweight and obesity in the UK in 2010. Br J Cancer. 2011;105 Suppl 2:S34-7.

15. Renehan AG, Soerjomataram I, Leitzmann MF. Interpreting the epidemiological evidence linking obesity and cancer: a framework for population-attributable risk estimations in Europe. Eur J Cancer. 2010;46:2581-92.

16. Song YM, Sung J, Ha M. Obesity and risk of cancer in postmenopausal Korean women. J Clin Oncol. 2008;26:3395-402.

17. Jee SH, Yun JE, Park EJ, Cho ER, Park IS, Sull JW, et al. Body mass index and cancer risk in Korean men and women. Int J
Cancer. 2008;123:1892-6.

18. Oh SW, Yoon YS, Shin SA. Effects of excess weight on cancer incidences depending on cancer sites and histologic findings among men: Korea National Health Insurance Corporation Study. J Clin Oncol. 2005;23:4742-54.

19. Lauby-Secretan B, Scoccianti C, Loomis D, Grosse Y, Bianchini F, Straif K, et al. Body fatness and cancer: viewpoint of the IARC Working Group. N Engl J Med. 2016;375:794-8.

20. World Cancer Research Fund; American Institue for Cancer Research. Food, nutrition, physical activity, and the prevention of cancer: a global perspective. Washington, DC: American Institue for Cancer Research; 2007.

21. World Cancer Research Fund; American Institue for Cancer Research. Continuous update project report: food, nutrition, physical activity, and the prevention of cancer. Washington, DC: American Institue for Cancer Research; 2007.

22. Cao Y, Stampfer M, Willett W, Spiegelman D, Manson J, Rimm $\mathrm{E}$, et al. Long-term aspirin use and total and cancer-specific mortality. Cancer Res. 2017;77(13 Suppl):Abstr 3012.

23. Cao Y, Nishihara R, Wu K, Wang M, Ogino S, Willett WC, et al. Population-wide impact of long-term use of aspirin and the risk for cancer. JAMA Oncol. 2016;2:762-9.

24. Chubak J, Whitlock EP, Williams SB, Kamineni A, Burda BU, Buist DS, et al. Aspirin for the prevention of cancer incidence and mortality: systematic evidence reviews for the U.S. Preventive Services Task Force. Ann Intern Med. 2016;164:814-25.

25. Hayes J, Richardson A, Frampton C. Population attributable risks for modifiable lifestyle factors and breast cancer in New Zealand women. Intern Med J. 2013;43:1198-204.

26. World Health Organization. The global burden of disease. Geneva: World Health Organization; 2007.

27. Statistics Korea. Korean statistical information service: population (estimated population, life table), welfare (causes of death, cancer registry). Daejeon: Statistics Korea; 2015.

28. Park E, Park J. The analysis and reduction strategies of cancer burden in Korea. Seoul: Korean Foundation for Cancer Research; 2012.

29. Jung KW, Won YJ, Oh CM, Kong HJ, Lee DH, Lee KH, et al. Cancer statistics in Korea: incidence, mortality, survival, and prevalence in 2014. Cancer Res Treat. 2017;49:292-305.

30. Reeves GK, Pirie K, Beral V, Green J, Spencer E, Bull D, et al. Cancer incidence and mortality in relation to body mass index in the Million Women Study: cohort study. BMJ. 2007;335:1134.

31. Bergstrom A, Pisani P, Tenet V, Wolk A, Adami HO. Overweight as an avoidable cause of cancer in Europe. Int J Cancer. 2001;91:421-30.

32. Arnold M, Pandeya N, Byrnes G, Renehan PA, Stevens GA, Ezzati PM, et al. Global burden of cancer attributable to high body-mass index in 2012: a population-based study. Lancet Oncol. 2015;16:36-46.

33. Park YJ, Gu BS, Kang HC, Chon SH, Yoon JW. The menopausal age and climacteric symptoms, and the related factors of Korean women. Korean J Women Health Nurs. 2001;7:473-85. 